\title{
Case Report: Vestibular Rehabilitation in Isolated Otolith Dysfunction After Covid-19: A Case Report
}

\author{
Parisa Jalilzadeh Afshari ${ }^{* *}$ (1) \\ 1. Department of Audiology, University of Social Welfare and Rehabilitation Sciences, Tehran, Iran.
}

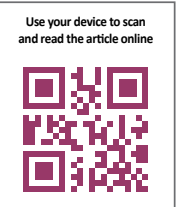

Citation: Jalilzadeh Afshari P. Vestibular Rehabilitation in Isolated Otolith Dysfunction After Covid-19: A Case Report. Iranian Rehabilitation Journal. 2021; 19(4):473-480. http://dx.doi.org/10.32598/irj.19.4.1392.1

http://dx.doi.org/10.32598/irj.19.4.1392.1

(c) (i) (8)

Article info:

Received: 14 Aug 2021

Accepted: 25 Oct 2021

Available Online: 01 Dec 2021

\section{Keywords:}

COVID-19, Vestibular system, Otolith dysfunction, Vestibular rehabilitation

\section{A B STRACT}

Introduction: A 56-year-old female with dizziness, imbalance, and a slight floating sensation was evaluated. Her symptoms started after infection with the novel coronavirus (SARS-CoV-2).

Case Presentation: Routine auditory test (pure tone audiometry), vestibular assessment (videonystagmography), and neurologic test results were in the normal range, but the otolith evaluations, such as cervical Vestibular Evoked Myogenic Potentials (cVEMP) and subjective visual vertical tests, showed abnormal findings. The patient underwent a ten-session individualized vestibular rehabilitation program (one session per week).

Results: After completing rehabilitation sessions, her chief complaints were alleviated, the performance on computerized posturography was improved, and the abnormal cVEMP amplitude asymmetry between ears disappeared.

Conclusion: In conclusion, vestibular disorders can happen after COVID-19 infection, presenting exclusively with isolated otolith dysfunction. In these patients, functional integrity assessment of the whole vestibular system is crucial, and vestibular rehabilitation may be beneficial. 


\section{Highlights}

- Vestibular disorders can happen after or during COVID-19 infections, presenting as isolated otolith dysfunction.

- The diagnosis of isolated otolith dysfunction could be achieved by vestibular evoked myogenic potentials and or subjective visual vertical tests.

- Vestibular rehabilitation is helpful for otolith disorders after COVID-19 infections.

\section{Plain Language Summary}

COVID-19 disease may cause serious respiratory problems and has an adverse impact on different parts of the body, including the balance system of the inner ear. The virus can specifically affect special parts of the inner ear called otolith organs which is one of the causes of dizziness, unsteadiness, and even falling. The otolith disorder may happen without any other balance problems and may be neglected without a comprehensive evaluation of the balance system. Vestibular rehabilitation and training can improve the balance function of patients with otolith disorder after the COVID-19 infection.

\section{Introduction}

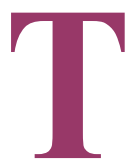

he novel Coronavirus 2019 (COVID-19) disease, a global pandemic, results from severe acute respiratory syndrome coronavirus 2 (SARS-CoV-2). The disease first appeared in China and then spread worldwide since 2019 [1,2]. The symptoms of the COVID-19 infection are diverse and nonspecific. Typical clinical symptoms are fever, sore throat, dry cough, shortness of breath, and weakness. Also, most patients may report myalgia, diarrhea, vomiting, and loss of taste and or smell. In severe infected cases, severe lung inflammation (pneumonia) with infiltrated changes on the chest CT scan develops and leads to patient hospitalization in an intensive care unit $[3,4]$. If the progression of the disease gets out of control, respiratory distress syndrome, septic shock, and coagulation dysfunction will be life-threatening [4]. The SARS-CoV-2 can also affect the peripheral and central nervous system and cause neurologic problems such as headache, facial palsy, unconsciousness, dizziness, encephalitis, and even stroke $[5,6]$. Some studies have shown that, like other viral infections, SARS-CoV-2 can damage the peripheral and central auditory-vestibular system by direct infection or vascular damage and have presentations, such as sensorineural hearing loss, tinnitus, and vertigo, occurring during or after the disease $[7,8]$. However, our knowledge about the possible clinical implications of COVID-19 and its management, especially in the vestibular system, is still limited [9-11]. In an article by Jafarzadeh (2021), the effects of vestibular rehabilitation were reported in 3 patients with SARS-CoV-2 infection. The cases showed abnormal caloric and video Head Impulse Test (vHIT) according to vestibular neuritis, and a homebased vestibular rehabilitation training was applied for management. Although this informal rehabilitation program positively impacted patients' symptoms, one case did not recover completely [9]. In this article, we report vestibular rehabilitation in a case with isolated otolith dysfunction after COVID-19.

\section{Case Presentation}

On April 10, 2021, a previously healthy 56-year-old woman was presented with dizziness and imbalance without nausea which increased with head movements and body motion, especially to the left side. She reported unsteadiness, floating, and the sensation of walking-on clouds during movements. In primary evaluation, her medical history revealed that about one month before the onset of vestibular complaints, she had a fever, sore throat, myalgia, headache, and dizziness without cough and shortness of breath. One day after the onset of symptoms, she was referred to the COVID-19 emergency ward, and a Reverse Transcriptase-Polymerase Chain Reaction (RT-PCR) test with nasopharyngeal swabs was performed for her. The RT-PCR was positive for SARSCov-2 infection, and the cycle threshold was 21 , which was an indicator of possible high levels of virus in her specimen. Her oxygen saturation was $98 \%$. Additional laboratory tests indexing inflammatory and coagulation cascades such as erythrocyte sedimentation rate, C-reactive protein, d-dimer, fibrinogen, and platelet count were within normal limits. After 48 hours, she no longer had a fever, and during the quarantine, her symptoms were alle- 
viated, but she continued to report dizziness. Despite the improvement in her general health at the end of the home quarantine, the dizziness intensified and was gradually associated with imbalance, especially during movement. She described her dizziness as feeling floating, lasting all the time, and worsened mainly by the movement. Due to the exacerbation of her dizziness, an Ear Nose Throat (ENT) specialist referred the patient to our Audiology Clinic in Rofeydeh Rehabilitation Hospital.

Her history revealed no hearing loss, balance disorder, tinnitus, and neurologic problems before the COVID-19 infection. The basic audiological assessments (audiometry and tympanometry) showed normal results. The hearing threshold of both sides was within normal limits, and type A was achieved in tympanometry. The diagnostic auditory brainstem response with click stimuli at supra-threshold levels was performed, and there was no sign of retro-cochlear involvement. The brain magnetic resonance imaging with and without contrast ruled out any neurologic findings. The bedside tests, including Romberg, gait, tandem gait, Fukuda, and past pointing, were all normal. Videonystagmography test battery (spontaneous nystagmus, oculomotor tests, positional and positioning tests, and caloric test) and head-shaking test were performed, and the results showed normal function without canal paralysis. The vestibulo-ocular gain for all semicircular canals in the video head impulse test (vHIT) was within normal limits, and there were no covert and overt saccades. The electrocochleography test result showed a normal summating potential/action potential (SP/AP) ratio in both ears.

The cervical vestibular evoked myogenic potentials (cVEMP) with $500 \mathrm{~Hz}$ alternating air conducted toneburst stimuli was monaurally performed for both ears. Briefly, cVEMP was recorded from the ipsilateral sternocleidomastoid muscle in the sitting position and head rotation to each side. The results demonstrated that the amplitude of the P13-N23 complex in the left ear decreased compared to the right ear, and the inter-aural amplitude asymmetry ratio was $60 \%$ (Figure 1 ). The subjective visual vertical (SVV) test was also applied. The patient was instructed to align a light bar vertically from a distance of $1 \mathrm{~m}$ in a dark room. The difference between the subject's vertical alignment and an actual vertical line was measured. Three trials were repeated for 10 conditions with and without visual conflict, and the final score was the average of the 3 trials for each condition (Table 1). The SVV was considered abnormal when the degree of deviation was greater than 2.5 [12] In our patient, the deviation of the light bar from vertical alignment was more than 2.5 , especially with leftward optokinetic visual conflict on the background (Table 1).

The active, reference, and ground electrodes were placed on the mid-portion of the Sternocleidomastoid (SCM) muscle, sternum, and forehead. The test was run
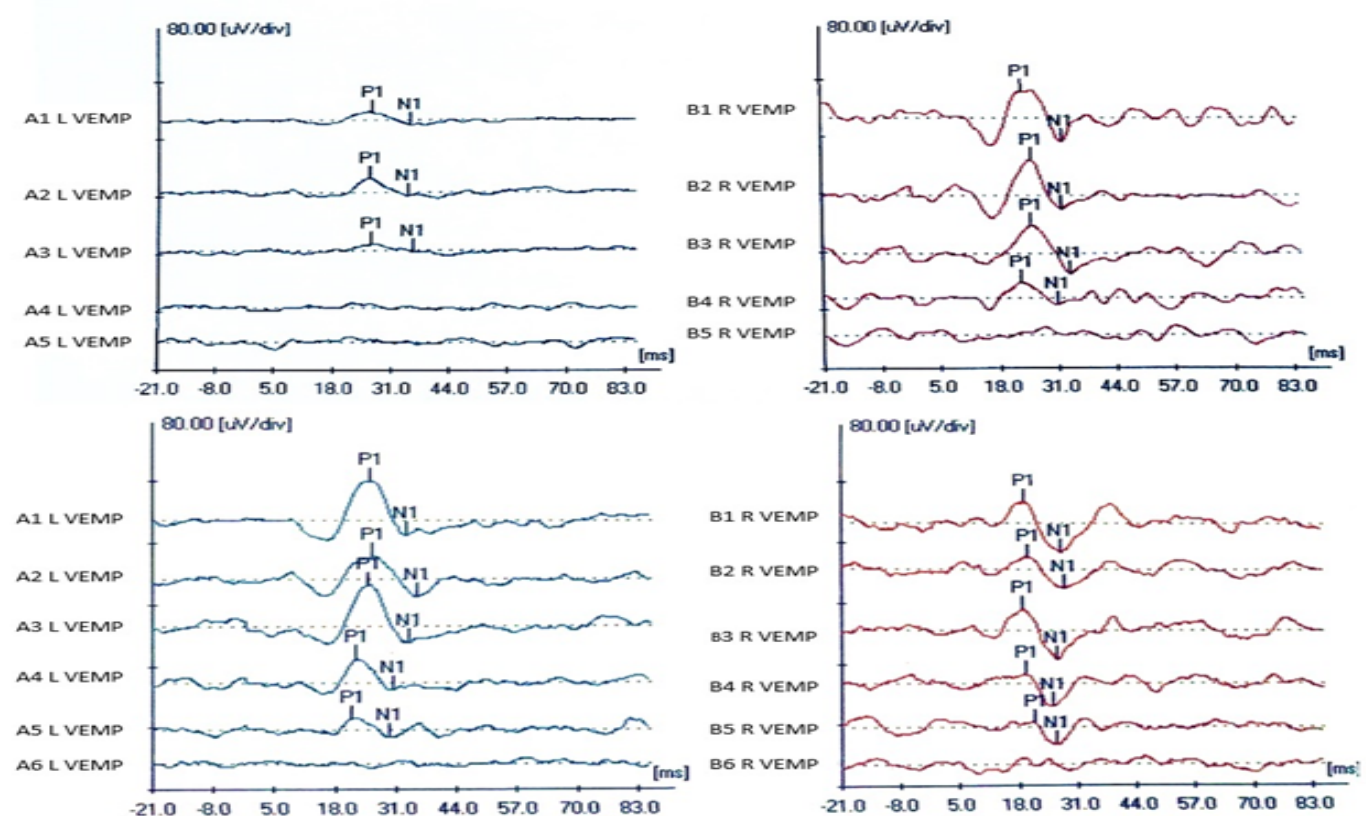

Iranian Rehabilitation Journal

Figure 1. The P13-N23 Complex of Cervical Vestibular Evoked Myogenic Potential (cVEMP) in the Left Ear (Left Panel) and Right Ear (Right Panel) Before (Upper Panel) and After (Lower Panel) Vestibular Rehabilitation 


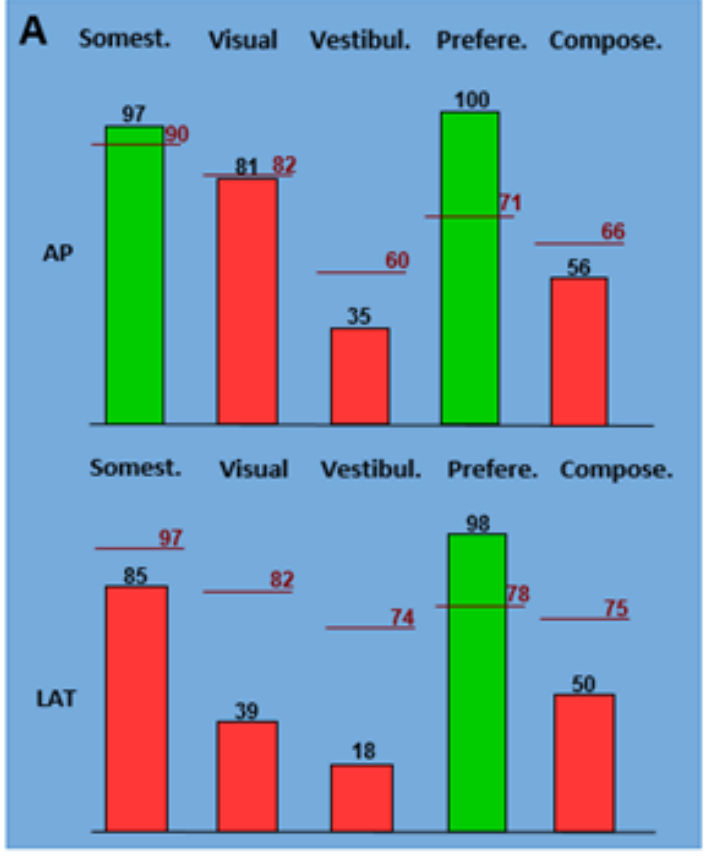

Figure 2. The Sensory Organization Test (SOT) results

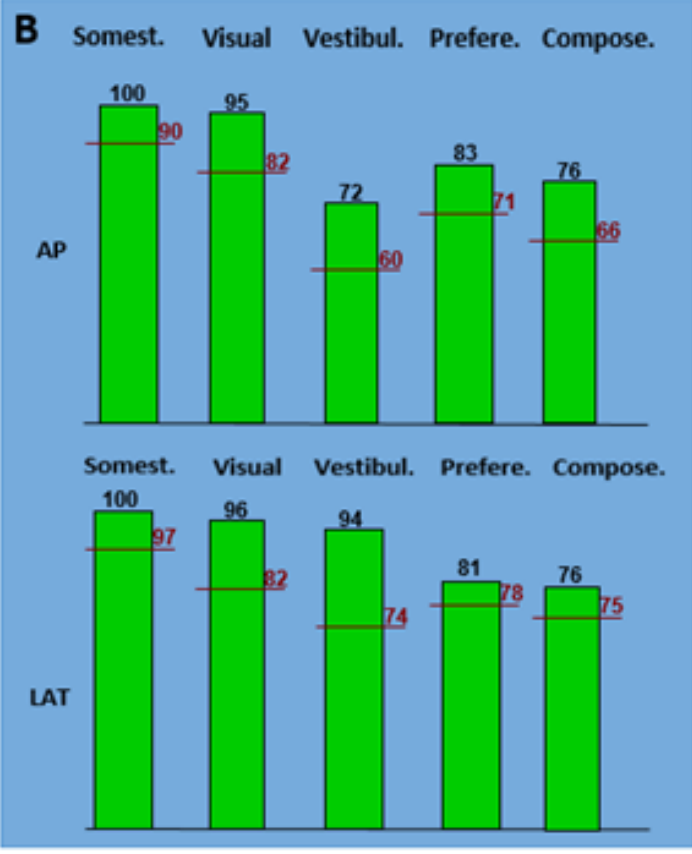

Iranian Rehabilitation Journal

A: The Left Panel Showing the Pre-rehabilitation Results and the Right Panel; B: Showing the Results After Vestibular Rehabilitation.

$\mathrm{AP}=$ Anterior-Posterior, $\mathrm{LAT}=$ Lateral.

firstly at $95 \mathrm{dBnHL}$; then, the intensity was decreased in 5-dB steps to determine the cervical Vestibular Evoked Myogenic Potentials (cVEMP) threshold.

Based on the patient's chief complaint, her performance was assessed on computerized posturography. The Sensory Organization Test (SOT), limits of stability, and static and dynamic vestibular stimulation tests were administered, too. The SOT assesses the individual's ability to integrate and use visual, proprioceptive, and vestibular cues for postural control. The test is done under 6 conditions (normal vision, fixed support absent vision, fixed support sway-referenced vision, fixed support normal vision, sway-referenced support absent vision, sway-referenced support sway-referenced vision, swayreferenced support), and the body oscillation is evaluated. In the absence conditions of somatosensory and visual cues, the subject must rely merely on vestibular information to prevent falling. The patient with vestibular involvement shows abnormal results on these conditions. The SOT scores of the current patient showed visual, vestibular, and composite scores below the normal range. The preferences result on SOT were $98 \%-100 \%$, demonstrating the patient's dependency on other sensory cues except than vestibular system (Figure 2). Limits of stability of posturography evaluate the furthest positions from the patient's center of pressure. Her stability limits showed abnormal distribution in different directions (front, back, right, and left) than the reference surface (Figure 3). To increase the sensitivity of static and dynamic postural control, the head movements in different planes (sagittal, frontal, horizontal) are superimposed, further stimulating the vestibular system. The balance control, despite a significant vestibular stimulation, is more challenging. The static and dynamic vestibular stimulation tests were done around the sagittal, frontal, and horizontal axis for her, and abnormal results were obtained (Figure 4).

The somatosensory score shows the patient's ability to use somatosensory input. The visual score represents the patient's ability to use visual cues for maintaining balance. The vestibular score shows the patient's ability to use vestibular cues when the visual and somatosensory cues are absent. A preferential score makes it possible to evaluate the ability to ignore erroneous visual information. The composite score is a general assessment of the patient's ability to use sensory information to keep balance. The outer circle represents the total surface, and the inner circle represents the reference surface of the 


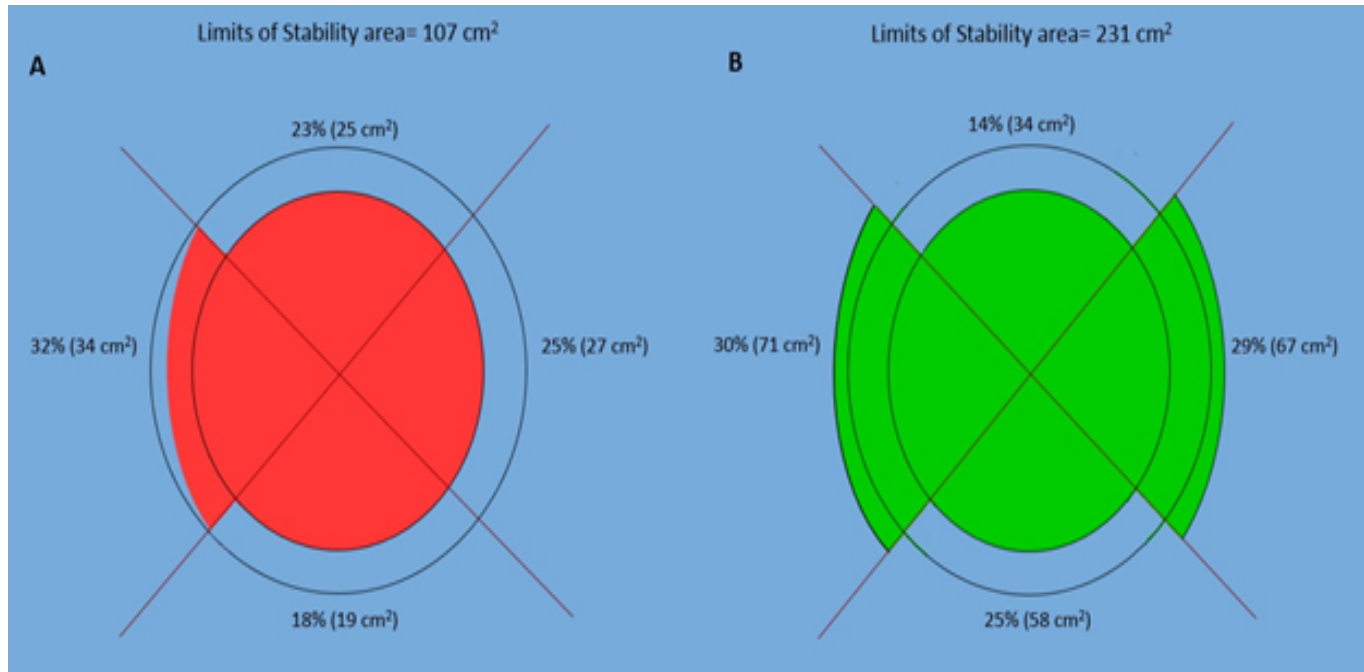

Figure 3. Limits of stability with eyes open before and after the vestibular rehabilitation

Iranian Rehabilitation Dourna

limits of the stability diagram. If the total surface is less than the reference surface, the circle appears red, otherwise green.

Once the patient signed the informed consent form, according to the mentioned findings, she was given individualized vestibular rehabilitation sessions for 10 weeks. The rehabilitation consisted of stabilization, weight-bearing, weight shift, postural control, adapta- tion, and habituation exercises. Besides, general balance training has been advised for home. The training difficulty was progressively and adaptively increased throughout the rehabilitation sessions based on the patient's performance. The exercises became harder by decreasing the needed time to complete the games, inserting foam on the posturography platform to reduce the somatosensory cues, and movement of the visual background in response to the patient's movement. Af-
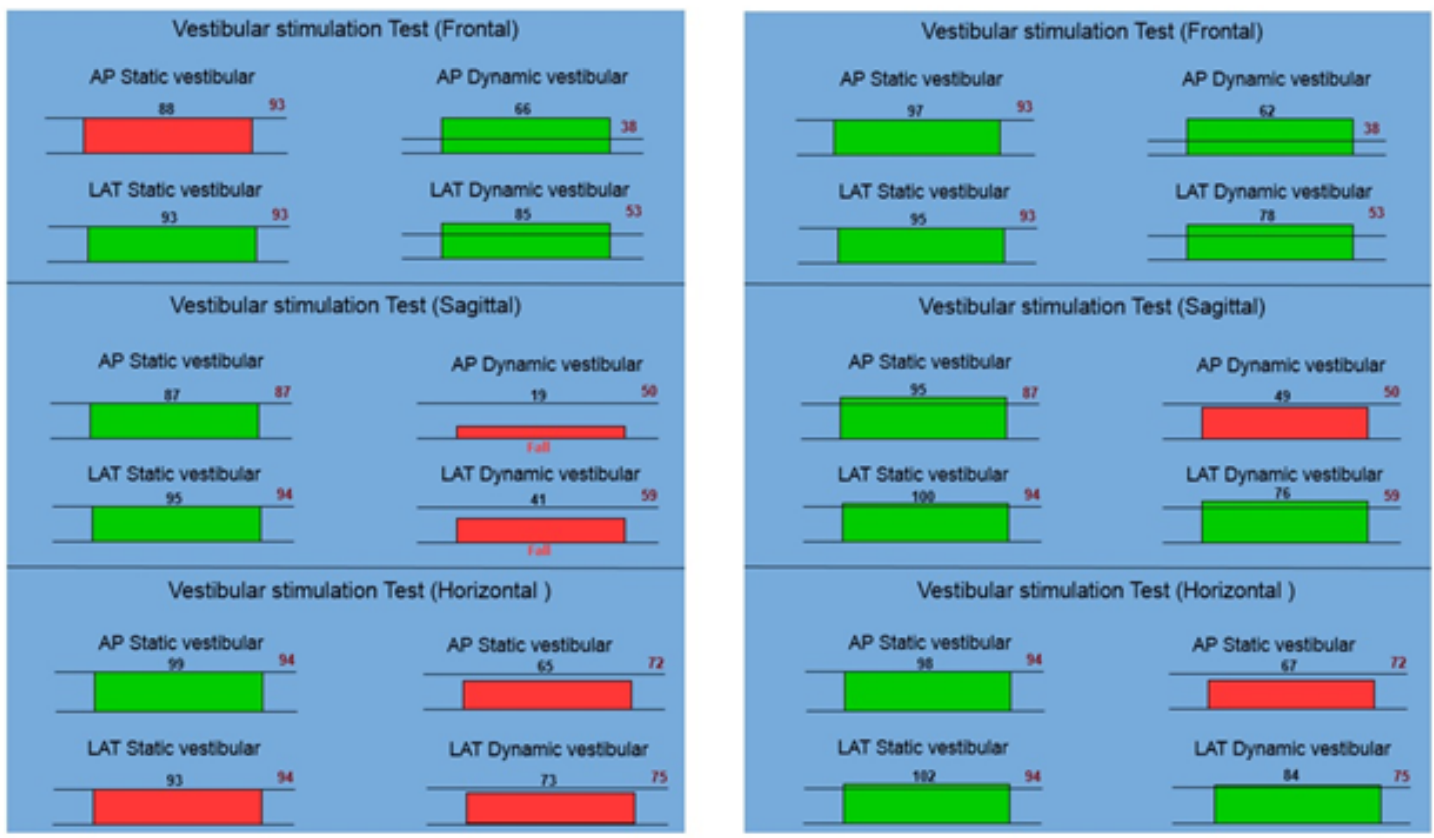

Пranian Rehabilitation \ournal

Figure 4. Vestibular Stimulation Tests Scores in static and dynamic platform with head movement in frontal, sagittal, and horizontal planes before (left panel) and after (right panel) the vestibular rehabilitation

$\mathrm{AP}=$ Anterior-Posterior, $\mathrm{LAT}=$ Lateral. 
Table 1. Subjective Visual Vertical (SVV) test results before and after vestibular rehabilitation, the final scores are the average of 3 trials in each condition

\begin{tabular}{|ccc|}
\hline SVV results & Pre-rehabilitation Score & Post-rehabilitation Score \\
\hline Normal pattern (light bar alignment from right) & -1.3 & 0.9 \\
\hline Normal pattern (light bar alignment from left) & -3.1 & -1.2 \\
\hline $\begin{array}{c}\text { Twenty degrees rightward optokinetic back-ground conflict } \\
\text { pattern (light bar alignment from right) }\end{array}$ & 2.2 & -0.7 \\
\hline $\begin{array}{c}\text { Twenty degrees rightward optokinetic back-ground conflict } \\
\text { pattern (light bar alignment from left) }\end{array}$ & -0.8 & 1.4 \\
\hline $\begin{array}{c}\text { Twenty degrees leftward optokinetic back-ground conflict } \\
\text { pattern (light bar alignment from right) }\end{array}$ & -4.1 & -1.9 \\
\hline $\begin{array}{c}\text { Twenty degrees leftward optokinetic back-ground conflict } \\
\text { pattern (light bar alignment from left) }\end{array}$ & -6.3 & -3.2 \\
\hline $\begin{array}{c}\text { Forty degrees rightward optokinetic back-ground conflict } \\
\text { pattern (light bar alignment from right) }\end{array}$ & 0.3 & 0.5 \\
\hline $\begin{array}{c}\text { Forty degrees rightward optokinetic back-ground conflict } \\
\text { pattern (light bar alignment from left) }\end{array}$ & -1.2 & -1.5 \\
\hline $\begin{array}{c}\text { Forty degrees leftward optokinetic background conflict pat- } \\
\text { tern (light bar alignment from right) }\end{array}$ & -3.6 & -0.8 \\
\hline $\begin{array}{c}\text { Forty degrees leftward optokinetic background conflict pat- } \\
\text { tern (light bar alignment from left) }\end{array}$ & -4.9 & -1.6 \\
\hline
\end{tabular}

Пranian Rehabilitation Journal

ter completing the rehabilitation program, the vestibular tests were repeated, and normal results were obtained. The re-administration of computerized posturography showed normal findings after rehabilitation, including improved SOT (Figure 2), limits of stability (Figure 3), and vestibular stimulation tests scores (Figure 4). It is obvious that the sensory preference was decreased due to improvement in visual and vestibular responses. The patient's cVEMP results following vestibular rehabilitation were within normal limits. There was no inter-aural asymmetry between the cVEMP amplitudes of the P13N23 complex (Figure 1). Also, vestibular rehabilitation improved SVV test results. The deviation degree of the light bar in vertical alignment was decreased specifically in challenging test conditions with optokinetic visual conflict on the background (Table 1).

\section{Discussion}

COVID-19 may suddenly or progressively cause dizziness and or vertigo in individuals without previous vestibular problems. A few studies on this topic show that infection with SARS-CoV-2 may accompany some vestibular symptoms. Although the exact mechanism is unclear, the probable causes of balance or vestibular disorders are as follows: direct invasion of the virus to the inner ear, involvements of the central vestibular system and its connections, hypoxia, vascular changes like vasculitis, and coagulation events $[13,14]$. Balance/ vestibular disorders caused by COVID-19 can be seen in the acute phase of the disease or after recovery [15] The association of dizziness with other important manifestations such as headache, blurred vision, numbness, unconsciousness, and so on must be taken seriously.

The clinical significance of balance disorders in SARSCoV-2 infection has been reported in a study using computerized dynamic posturography (CDP) and the vestibular evoked myogenic potential (VEMPs). The study showed that COVID-19 could involve the peripheral and or central vestibular system leading to abnormal findings in CDP and VEMPs, which may occur even after the patient's recovery from infection [16].

The present article reports a case with balance problems after COVID-19 infection. Her comprehensive assessment of the vestibular system showed abnormal findings in cVEMP, SVV, and also computerized posturography, which suggest isolated otolith dysfunction as a result of the otolith, vestibular nerve, or their central connections involvement. Thus, viral activation of SARS-CoV-2 infection can affect only a specific part of the vestibular system without necessarily involving other parts. The isolated otolith dysfunction was first described as otolith vertigo by Murofushi et al. [17]. Specific dysfunction of the utricle and or saccule may be possible and cause symptoms such as body tilt sensation, swaying, floating, walking on pillows, room tilt illusion, and even feeling of falling [18]. In the literature, there are few studies with subjects who were diagnosed with isolated otolith 
disorders, and the reported prognosis was controversial. Previous research suggested that otolith disorders have a poorer prognosis [19]. However, other studies reported the recovery of VEMP responses in patients with otolith dysfunctions $[16,17]$.

The important observation, in this case, was the disabling effects of isolated otolith disorder which adversely affected her balance performance on computerized posturography. This observation supports a study result that individuals with the absent response on VEMP have poorer balance [19]. It seems that isolated otolith organ involvement can adversely affect subjective and objective clinical assessments of balance function, found in both animals and human studies [19]. This finding shows the importance of otolith function evaluation in individuals with vestibular disorder symptoms.

The vestibular rehabilitation in this particular case showed improvement in cVEMP, SVV, and computerized posturography. This finding is in line with a study by Jafarzadeh in which the home-based vestibular rehabilitation improved vestibular dysfunction in 3 patients with vestibular neuritis after COVID-19 infection [9]. Also, the obtained result of the current study is in line with a previous study in which the cervical and ocular VEMP results of a patient with isolated otolith disorder showed recovery [16]. Although the exact cellular or neuronal pathways of otolith recovery are unknown, it seems that vestibular rehabilitation has benefits even for isolated otolith dysfunction and leads to the general improvement of balance function. The positive consequences of vestibular rehabilitation affect the overall integration of all vestibular sensory information.

In conclusion, SARS-CoV-2 infection may cause vestibular symptoms proven by the abnormal otolith function tests, despite normal responses in other vestibular tests. Exclusively abnormal cVEMP or SVV must be considered as an isolated otolith disorder which may be a distinct cause of dizziness. Vestibular rehabilitation can be helpful for isolated otolith dysfunction.

\section{Ethical Considerations}

\section{Compliance with ethical guidelines}

There were no ethical considerations to be considered in this research. Written consent has been obtained from the subject.

\section{Funding}

This research did not receive any grant from funding agencies in the public, commercial, or non-profit sectors.

\section{Conflict of interest}

The authors declared no conflict of interest.

\section{Acknowledgments}

The author gratefully acknowledges the Clinical Research Development Center of Rofeideh Rehabilitation Hospital for constant support during this research.

\section{References}

[1] Wang C, Horby PW, Hayden FG, Gao GF. A novel coronavirus outbreak of global health concern. The Lancet. 2020 395(10223):470-3. [DOI:10.1016/S0140-6736(20)30185-9]

[2] Zhu N, Zhang D, Wang W, Li X, Yang B, Song J, et al. A novel coronavirus from patients with pneumonia in China, 2019. The New England Journal of Medicine. 2020; 382(8):72733. [DOI:10.1056/NEJMoa2001017] [PMID] [PMCID]

[3] Huang C, Wang Y, Li X, Ren L, Zhao J, Hu Y, et al. Clinical features of patients infected with 2019 novel coronavirus in Wuhan, China. The Lancet. 2020; 395(10223):497-506. [DOI:10.1016/S0140-6736(20)30183-5]

[4] She J, Jiang J, Ye L, Hu L, Bai C, Song Y. 2019 novel coronavirus of pneumonia in Wuhan, China: Emerging attack and management strategies. Clinical and Translational Medicine. 2020; 9(1):19. [DOI:10.1186/s40169-020-00271-z] [PMID] [PMCID]

[5] Iadecola C, Anrather J, Kamel H. Effects of COVID-19 on the nervous system. Cell. 2020; 183(1):16-27.e1. [DOI:10.1016/j. cell.2020.08.028] [PMID] [PMCID]

[6] Ahmad I, Rathore FA. Neurological manifestations and complications of COVID-19: A literature review. Journal Of Clinical Neuroscience : Official Journal of the Neurosurgical Society of Australasia. 2020; 77:8-12. [DOI:10.1016/j. jocn.2020.05.017] [PMID] [PMCID]

[7] Fancello V, Hatzopoulos S, Corazzi V, Bianchini C, Skarżyńska MB, PelucchiS, etal.SARS-CoV-2 (COVID-19) and audio-vestibular disorders. International Journal of Immunopathology and Pharmacology. 2021; 35:20587384211027373. [DOI:10.1177/20587384211027373] [PMID] [PMCID]

[8] Giannantonio S, Scorpecci A, Montemurri B, Marsella P. Case of COVID-19-induced vestibular neuritis in a child. BM Case Reports. 2021; 14(6):e242978. [DOI:10.1136/bcr-2021242978] [PMID] [PMCID]

[9] Jafarzadeh S. Vestibular rehabilitation in three patients with COVID-19 induced vestibular neuritis. Journal of Rehabilitation Sciences \& Research. 2021. https://jrsr.sums.ac.ir/article_47843.html 
[10] Malayala SV, Mohan G, Vasireddy D, Atluri P. A case series of vestibular symptoms in positive or suspected COVID-19 patients. Le Infezioni in Medicina. 2021; 29(1):117-22. [PMID]

[11] Mat Q, Noël A, Loiselet L, Tainmont S, Chiesa-Estomba CM, Lechien JR, et al. Vestibular neuritis as clinical presentation of COVID-19. 2021; 145561321995021. [DOI:10.1177/0145561321995021] [PMID]

[12] Brandt T. Vertigo: its multisensory syndromes. New York: Springer New York; 2013. https:/ / www.google.com/books/ edition/Vertigo/HCwLCAAAQBAJ?hl=en\&gbpv=0

[13] Wu Y, Xu X, Chen Z, Duan J, Hashimoto K, Yang L, et al. Nervous system involvement after infection with COVID-19 and other coronaviruses. Brain, Behavior, and Immunity. 2020; 87:18-22. [DOI:10.1016/j.bbi.2020.03.031]

[14] Ralli M, Campo F, Angeletti D, Minni A, Artico M, Greco $\mathrm{A}$, et al. Pathophysiology and therapy of systemic vasculitides. EXCLI Journal. 2020; 19:817-54. [PMID]

[15] Amiri M, Hasanalifard M, Delphi M. Impact of COVID-19 on the auditory and vestibular system. Auditory and Vestibular Research. 2021; 30(3):152-9. [DOI:10.18502/avr.v30i3.6528]

[16] Yılmaz O, Mutlu BÖ, Yaman H, Bayazıt D, Demirhan $\mathrm{H}$, Bayazit YA. Assessment of balance after recovery from Covid-19 disease. Auris, Nasus, Larynx. 2021; S03858146(21)00233-9. [DOI:10.1016/j.anl.2021.08.011] [PMCID]

[17] Murofushi T, Iwasaki S, Ushio M. Recovery of vestibular evoked myogenic potentials after a vertigo attack due to vestibular neuritis. Acta Oto-Laryngologica. 2006; 126(4):364-7. [PMID]

[18] Park HG, Lee JH, Oh SH, Park MK, Suh MW. Proposal on the diagnostic criteria of definite isolated otolith Dysfunction. Journal Of Audiology \& Otology. 2019; 23(2):103-11. [DOI:10.7874/jao.2018.00374] [PMID] [PMCID]

[19] Murray KJ, Hill KD, Phillips B, Waterston J. The influence of otolith dysfunction on the clinical presentation of people with a peripheral vestibular disorder. Physical Therapy. 2007; 87(2):143-52. [DOI:10.2522/ptj.20060004] [PMID] 